Clinical Medicine

Poster

Abstract ID: 67

\title{
Preoperative Acetate Templeting in Primary Total Knee Replacement: Does it Matter?
}

Khairul Nizam Siron | Mohd Shahidan Noor Rahin | Aminuddin Che Ahmad | Ahmad Hafiz Zulkifly

Department of Orthopaedics, Traumatology \& Rehabilitation, Kulliyyah of Medicine, International Islamic University Malaysia

Introduction: Preoperative templating is a crucial step in a total knee arthroplasty. We conducted a prospective study of 100 patients subjected for total knee arthroplasty. The aim of this study is to determine whether the size of the prostheses used in primary knee arthroplasty may be reliable and accurately predicted using acetate templating techniques. Methods: A cohort of 100 patients plan for Total Knee Arthroplasty was included in this cross-sectional study. Pre-operative radiographs were templated using acetate templates (Zimmer, Warsaw, Indiana, USA). The mismatch between templated and implanted sizes were observed and recorded. The accuracy and reliability of preoperative templating were assessed and analyzed using student t-test and ANOVA. Results: Using the acetate template technique, it was possible to accurately predict the tibial component size in $48 \%$ and femoral component in $47 \%$ of the knees. When the accuracy to within one size was included, the templating was correct in $95 \%$ and $97 \%$ respectively. There was no size mismatch for more than 2 sizes. This study has shown a higher tendency to template smaller size for both components in total knee arthroplasty. Conclusions: We conclude that the acetate templating method is reliable method in determining and guiding the surgeon to predict for varies prostheses sizes preoperatively in centre where the digital template facility is not available.

KEYWORDS: total knee arthroplasty, templating 